\title{
Ecophylogeny of the endospheric root fungal microbiome of co-occurring Agrostis stolonifera
}

\author{
Amandine Lê Van ${ }^{1}$, Achim Quaiser ${ }^{1}$, Marie Duhamel ${ }^{1,2}$, Sophie Michon-Coudouel ${ }^{3}$, Alexis Dufresne ${ }^{1}$, \\ Philippe Vandenkoornhuyse ${ }^{\text {Corresp. } 1}$ \\ 1 CNRS, UMR6553 Ecobio, Université de Rennes 1, Rennes, France \\ 2 Department of Biology, Stanford University, Stanford, California, United States \\ 3 CNRS, UMS3343 OSUR, Université de Rennes 1, Rennes, France \\ Corresponding Author: Philippe Vandenkoornhuyse \\ Email address: philippe.vandenkoornhuyse@univ-rennes1.fr
}

Background. Within the root endosphere, fungi are known to be important for plant nutrition and resistance to stresses. However, description and understanding of the rules governing community assembly in the fungal fraction of the plant microbiome remains scarce.

Methods. We used an innovative DNA- and RNA-based analysis of co-extracted nucleic acids to reveal the complexity of the fungal community colonizing the roots of an Agrostis stolonifera population. The normalized RNA/DNA ratio, designated the 'mean expression ratio', was used as a functional trait proxy. The link between this trait and phylogenetic relatedness was measured using the Blomberg's $K$ statistic.

Results. Fungal communities were highly diverse. Only $\sim 1.5 \%$ of the 635 OTUs detected were shared by all individuals, however these accounted for $33 \%$ of the sequence number. The endophytic fungal communities in plant roots exhibit phylogenetic clustering that can be explained by a plant host effect acting as environmental filter. The 'mean expression ratio' displayed significant but divergent phylogenetic signals between fungal phyla.

Discussion. These results suggest that environmental filtering by the host plant favours the coexistence of related and similar OTUs within the Basidiomycota community assembly, whereas the Ascomycota and Glomeromycota communities seem to be impacted by competitive interactions which promote the co-existence of phylogenetically related but ecologically dissimilar OTUs. 
1

2

3

4 Amandine Lê Van ${ }^{1}$, Achim Quaiser ${ }^{1}$, Marie Duhamel ${ }^{1,2}$, Sophie Michon-Coudouel ${ }^{3}$, Alexis

5 Dufresne $^{1}$, Philippe Vandenkoornhuyse ${ }^{1}$

6

$7 \quad{ }^{1}$ Université de Rennes 1, CNRS, UMR 6553 Ecobio, campus Beaulieu, 35000 Rennes (France)

$8 \quad{ }^{2}$ Stanford University, Department of Biology, Stanford, 94305 CA (United-States)

11 Author for correspondence:

12

${ }^{3}$ Université de Rennes 1, CNRS, UMS 3343 OSUR, campus Beaulieu, 35000 Rennes (France)

Prof. Philippe Vandenkoornhuyse

Tel: +33 -2 23235007

Email: philippe.vandenkoornhuyse@univ-rennes1.fr

Running title: The root fungal microbiome

\section{$(7$}

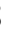
19 


\section{Summary}

Background. Within the root endosphere, fungi are known to be important for plant nutrition and resistance to stresses. However, description and understanding of the rules governing community assembly in the fungal fraction of the plant microbiome remains scarce.

Methods. We used an innovative DNA- and RNA-based analysis of co-extracted nucleic acids to reveal the complexity of the fungal community colonizing the roots of an Agrostis stolonifera population. The normalized RNA/DNA ratio, designated the 'mean expression ratio', was used as a functional trait proxy. The link between this trait and phylogenetic relatedness was measured using the Blomberg's K statistic.

Results. Fungal communities were highly diverse. Only $\sim 1.5 \%$ of the 635 OTUs detected were shared by all individuals, however these accounted for $33 \%$ of the sequence number. The endophytic fungal communities in plant roots exhibit phylogenetic clustering that can be explained by a plant host effect acting as environmental filter. The 'mean expression ratio' displayed significant but divergent phylogenetic signals between fungal phyla.

Discussion. These results suggest that environmental filtering by the host plant favours the coexistence of related and similar OTUs within the Basidiomycota community assembly, whereas the Ascomycota and Glomeromycota communities seem to be impacted by competitive interactions which promote the co-existence of phylogenetically related but ecologically dissimilar OTUs. 


\section{Introduction}

Plants are colonized by a wide collection of microorganisms forming the plant microbiome. The plant microbiome supports additive functions involved in the plant's adjustments to environmental conditions (for review, Vandenkoornhuyse et al., 2015), and thus controls, in part, plant fitness. Understanding the composition and rules of assembly within the plant microbiome is currently a key question in ecology.

Plant roots can be considered as a well-delimited ecological compartment with the endosphere constituting a 'restricted area' (Vandenkoornhuyse et al., 2015). Microbial communities within this compartment differ markedly in their composition from both the surrounding rhizospheric and soil microbial communities (e.g. Bulgarelli et al., 2012; Schlaeppi et al., 2014; Fonseca-García et al., 2016). Beside the well-known mycorrhizal fungi, nitrogenfixing bacteria, and plant-growth-promoting bacteria (PGPB) the plant microbiome includes a high diversity of associated microorganisms (e.g. Vandenkoornhuyse et al., 2002a; Lambais et al., 2006; Lundberg et al., 2012; Bulgarelli et al., 2012; Peiffer et al., 2013; Schlaeppi et al., 2014). The coexistence of numerous microbial taxa in plant roots is striking and raises the question of the assembly rules underlying these complex communities. However, this aspect remains poorly understood.

To date, most microbial ecology studies have addressed species diversity without taking into account the phylogenetic relatedness among microorganisms. However, our understanding of community assembly can be significantly improved by studying the community's phylogenetic structure (Webb et al., 2002). Assuming that related species share more similar traits than distant species, an analysis of phylogenetic structure can be used to link phylogenetic patterns to ecological processes (Cavender-Bares et al., 2009). According to the niche-based theory, specific 
phylogenetic patterns can be generated both by inter-specific interactions and environmental filtering by the host plant (e.g. Helmus et al., 2007; Cavender-Bares et al., 2009). In the phylogenetic pattern of overdispersion, species are less related to each other than species assembled at random from a regional pool. This pattern can be promoted, for example, by competition among related species which is expected to limit similarity (Diamond 1975; Cahill et al., 2008). Similarly, facilitative interactions are known to increase phylogenetic diversity in plant communities when facilitation occurs among distantly related species and favours species overdispersion (Valiente-Banuet \& Verdú, 2007). These mechanisms are counterbalanced by environmental filtering that selects for similar traits (Mayfield \& Levine, 2010) and may promote phylogenetic clustering. In a clustered phylogenetic pattern, species within the community are more related to each other than expected. Conversely, an absence of phylogenetic structure indicates that the species within the community are a random assemblage of the regional species pool. To be able to draw inferences from phylogenetic structure, the main assumption that phylogenetic relatedness is linked to ecological similarity, must be respected (Kembel, 2009). This assumption is satisfied when a phylogenetic signal can be measured. The phylogenetic signal is a metric used to measure this link by comparing trait similarity to phylogenetic relatedness (Blomberg et al., 2003).

Plants are sessile organisms that have to cope with the environmental changes they experience. One recent idea is that microorganism recruitment within the plant microbiome allows these changes to be buffered (Vandenkoornhuyse et al., 2015). Some filtering by the host plant can therefore be expected and should leave a specific signature in the phylogenetic structure. In this study, the fungal microbiome was defined by analyzing $18 \mathrm{~S}$ rRNA amplicon sequences produced from co-extracted DNA and RNA from plant root samples to identify the metabolically active 
89

microbial community (Klein et al., 2016). These co-extracted DNA- and RNA-based data were used to compare the endospheric fungal community composition and diversity and the core microbiome amongst 19 co-occurring Agrostis stolonifera plants. We introduced the 'mean expression ratio' as a proxy for functional trait, a functional trait being a measure related to species (i.e. Operational Taxonomic Units, OTUs hereafter) in ecological terms (i.e. activity, interactions) (Diaz \& Cabibo, 2001). In microbial communities these traits are for example, the ability to colonize plant roots, the metabolic activity at different temperatures, or the ability to fix nitrogen. The 'mean expression ratio' allows each OTU to be described by integrating the information produced from either the DNA or the RNA (i.e. ratio of observed number of sequences for each OTU). Under the hypothesis that traits favourable to an endophytic life style are phylogenetically conserved (Martiny et al., 2013), we could expect that the 'mean expression ratio' of related microbial OTUs in plants would be more similar than that of phylogenetically distant OTUs. We investigated these hypotheses by (i) analyzing the phylogenetic structure of 19 endophytic fungal communities from Agrostis stolonifera roots (ii) searching for a phylogenetic signal using the 'mean expression ratio' and, (iii) testing whether the phylogenetic signal was conserved among the main fungal phyla. We limited possible stochastic effects by using spatially aggregated host plants.

\section{Materials and methods}

\section{Plant harvesting and root sampling}

Turf samples (L44 x W34 x D23 cm) were collected from a peatland in the Parc Naturel Régional des Marais du Cotentin et du Bessin, Normandy, France (49.284656-1.393090). The 
113 sampled turf vegetation was dominated by Agrostis stolonifera ( $\sim 94 \%$ coverage), a generalist

114 plant often found in disturbed habitats, wetland margins and fields. The other co-occurring plants

115 were Potentilla anserina, Mentha spp. and Hydrocotyle vulgaris. Turfs were placed in a growth

116 chamber for four months at $15^{\circ} \mathrm{C}$ with $16 \mathrm{~h}$ light per day, and $80 \%$ humidity to avoid stochastic

117 environmental effects. Nineteen co-occurring Agrostis stolonifera plants were manually

118 harvested from a single turf. During sampling, the turf was divided into 3 disconnected blocks

119 but as there was no block effect in the observed variances, all data were pooled for the analyses.

120 The sampled plants were of similar age with comparable root systems. No difference in the

121 phenotypic traits of sampled plants was noticed. The roots were washed with tap water to remove

122 soil and the surface roots were sterilized by washing three times with $0.1 \%$ Triton $\mathrm{X}-100$ and

123 rinsed five times with sterile distilled water (Vandenkoornhuyse et al., 2007). The roots were

124 then transferred into RNAlater (Sigma-Aldrich) and stored at $-20^{\circ} \mathrm{C}$.

125

126

127

128

129

130

131

132

133

134

135

136

\section{Nucleic acid extraction and $18 S$ rRNAamplicon sequencing}

These sampled roots were crushed into powder with a mortar and pestle in liquid nitrogen. RNA and DNA were co-extracted from the nineteen samples using a modified RNeasy Plant Mini kit (Qiagen) protocol. The on-column DNase digestion was skipped to keep the DNA in the total extracts. For RNA extraction half of the total nucleic extracts were treated with RQ1 DNAse (Promega) and the complete elimination of DNA was confirmed by $18 \mathrm{~S}$ rRNA targeting PCR. RNA and DNA quality was checked using the RNA 6000 Pico kit or the High sensitivity DNA kit, respectively, on a 2100 Bioanalyzer (Agilent). PCRs were performed using the primer pair SSU0817 (5'-TTAGCATGGAATAATRRAATAGGA-3') -NS22B (5'AATTAAGCAGACAAATCACT-3') targeting a region of about $530 \mathrm{bp}$ of the 18S rRNA gene that includes the variable regions V4 (partial) and V5 (Borneman \& Hartin, 2000). These 
137 particular primers were chosen among a variety of candidate primers after an in silico analysis,

138 using Primer Search (Rice et al., 2000). The chosen set could amplify 94\% of the available

139 fungal sequences in silico, with the exception of Microsporidia, and only $1.3 \%$ of Viridiplantae

140 in the Silva database 115 (Quast et al., 2013). PCR amplifications were performed with fusion

141 primers containing sequencing adaptors, multiplex identifiers (MID) and PCR primers (Table

142 S1). The DNA was amplified by performing direct PCRs on the total nucleic acid extracts using

143 illustra puReTaq Ready-To-Go PCR Beads (GE Healthcare). Two microliters of DNA template

144 (at $\sim 1 \mathrm{ng} . \mu \mathrm{L}^{-1}$ ) were used in a final volume of $25 \mu \mathrm{L}$ with $0.2 \mu \mathrm{M}$ concentrations of each primer.

145 The PCR cycling protocol consisted of 35 cycles of denaturation $\left(95^{\circ} \mathrm{C}\right.$ for $\left.30 \mathrm{sec}\right)$, annealing

$146\left(54^{\circ} \mathrm{C}\right.$ for $\left.30 \mathrm{sec}\right)$ and elongation $\left(72^{\circ} \mathrm{C}\right.$ for $\left.1 \mathrm{~min}\right)$ with an initial denaturation step $\left(95^{\circ} \mathrm{C}\right.$ for 4

$147 \mathrm{~min}$ ) and a final elongation step $\left(72^{\circ} \mathrm{C}\right.$ for $\left.7 \mathrm{~min}\right)$. Two independent PCRs representing technical

148 replicates were performed for each sample. The RNA was amplified by performing RT-PCRs

149 using the Titan One Tube RT-PCR kit (Roche Molecular Systems). The reaction was carried out

150 in a final volume of $50 \mu \mathrm{L}$ with $0.2 \mu \mathrm{M}$ of each primer. Reverse transcription $\left(42^{\circ} \mathrm{C}\right.$ for $\left.30 \mathrm{~min}\right)$

151 was followed by PCR amplification with an initial denaturation step at $94^{\circ} \mathrm{C}$ for 3 min, 38 cycles

152 of $30 \mathrm{sec}$ at $94^{\circ} \mathrm{C}, 30 \mathrm{sec}$ at $54^{\circ} \mathrm{C}$ and $45 \mathrm{sec}(+5 \mathrm{sec} /$ cycle from the 11 th to the 38 th cycle $)$ at

$15368^{\circ} \mathrm{C}$ and a final elongation at $68^{\circ} \mathrm{C}$ for $7 \mathrm{~min}$. Two independent RT-PCRs were performed for

154 each sample. The quality of the PCR products was checked on High Sensitivity DNA chips

155 (Agilent) and purified amplicons were quantified by spectrofluorometry using the Quant-iT

156 PicoGreen dsDNA Assay kit (Invitrogen). The libraries were pooled in equimolar amounts and

157 purified using the AMPure XP system (Beckman-Coulter). Any traces of concatemerized

158 primers were removed by subjecting the libraries to microelectrophoresis on a Caliper Labchip

159 XT instrument (Perkin Elmer). The libraries were amplified by emPCR using the GS FLX 
160 Titanium MV emPCR Kit with Lib-L chemistry and sequenced on a GS FLX+ sequencer 161 (Roche/454) following the manufacturer's instructions.

162

\section{Sequence analysis using a dedicated automated pipeline}

Quality trimming and filtering of amplicons, OTU identification, and taxonomic assignments were carried out with a combination of publicly available sequencing data analysis tools (Cutadapt, Mothur, Dnaclust) and in-house python scripts within a Galaxy instance at the Genouest platform (http://www.genouest.org/), as described elsewhere (Ciobanu et al., 2014, Nunes et al., 2015, Ben Maamar et al., 2015). Briefly, sequences shorter than 200 bp in length, with homopolymers longer than 8 bp or with ambiguous nucleotides, were removed from the dataset. Sequences containing errors in the MID or primer sequences were discarded. Chimeric sequences were eliminated using the chimera.uchime command of the Mothur tool suite. It is well known that both PCR and pyrosequencing can induce erroneous sequences (Shakya et al., 2013b) leading to poor diversity estimates. To improve sequence quality, two independent PCR reactions were performed for each sample and replicates were sequenced. Only sequences displaying $100 \%$ identity among these technical replicates were retained for subsequent analysis. Sequences were grouped into OTUs with a sequence identity threshold of $97 \%$. Consequently one OTU was defined by at least two identical sequences originating from technical replicates. The taxonomic affiliations of the sequences and OTUs were determined by comparison with the SILVA database 115 (Quast et al., 2013). Two OTUs, representing 1132 sequences, assigned to the Chloroplastida, were removed. The study accession number in the European Nucleotide Archive is PRJEB12655. 
184 The samples were normalised to 1288 sequences for analyses of alpha and beta diversity whereas

185 the full dataset was used to analyze the core microbiome in co-occurring plants within a single 186 population. All statistical analyses were performed in R ( R core team 2013) using the VEGAN

187 package (Oksanen et al., 2011). To check the sequencing depth, rarefaction curves were

188

189

190

191

192

193 computed using the rarefaction function. Alpha diversity and richness were estimated for each sample using Hill diversity numbers (Hill, 1973) and the Chao 1 index (Chao, 1984). Hill diversity numbers allow accurate comparison of species/OTU diversity across samples. The significance of the Hill diversity numbers depends on the value of the q parameter in the Hill formula. This parameter allows species to be weighted more or less equally. For $q=0$, OTUs or species are weighted equally and the Hill diversity is equal to the OTU richness while for $\mathrm{q}=1$, the Hill diversity corresponds to the Shannon diversity and for $q=2$, the Hill diversity is equal to the Simpson index. One community can be considered more diverse when all of its Hill diversity numbers are higher than those of the other communities. The Chao 1 index estimates the unseen diversity by taking rare OTUs into account. The alpha diversities and taxonomic compositions of the DNA and RNA fractions were compared using paired Student's t-tests or Wilcoxon rank-sum tests only when the alpha diversity values did not follow a normal distribution or were heteroscedastic.

Beta diversity was studied by using non-metric multidimensional scaling (NMDS) with the BrayCurtis dissimilarity matrix. Data were transformed using the square root and the Wisconsin double standardization implemented in the metaMDS function. Procrustes analysis was conducted on the NMDS scores to assess the concordance between the communities in the DNA and RNA fractions. Significance of the concordance was tested by permutation $(10,000)$ using the protest function (Peres-Neto \& Jackson, 2001). 
207 We defined the core microbiome as the proportion of OTUs shared by the studied co-occurring 208 A. stolonifera plants. To determine whether the number of shared OTUs was dependent on the sampling effort, we performed random re-sampling and increased the sampling size from one to the total number of samples for each fraction. We defined the 'DNA core' and the 'RNA core' as the proportion of OTUs present in the DNA and the RNA fraction respectively of all samples.

\section{Phylogenetic tree construction}

Representative sequences of OTUs were aligned using SINA aligner v1.2.11 (Pruesse et al., 2012), imported from the non-redundant SILVA SSU Ref ARB database (release 115).

Alignments of the reference sequences and of representative OTU sequences were exported from ARB (Ludwig et al., 2004). Gaps and ambiguously aligned positions were excluded. One alignment was obtained for each main fungal phylum. The model of sequence evolution that best fitted the aligned sequences was selected using jModelTest v2.1.4 (Darriba et al., 2012). Phylogenetic trees were constructed by maximum likelihood using TREEFINDER and by bayesian inferences using MrBayes v.3.2.2 (Ronquist et al., 2012). Maximum likelihood bootstrap values were calculated from 1,000 replicates and Bayesian posterior probabilities were calculated using 100,000 generations sampled every 100 generations.

\section{Phylogenetic structure}

The community phylogenetic structure was studied by applying the Picante package (Kembel et al., 2010) to the global phylogenetic tree including all the fungal OTUs. Comparison of the phylogenetic relatedness of the OTUs in the DNA and RNA fractions of each sample with the local pool of OTUs (OTUs found in all samples) was based on two indices: the mean pairwise 
231 distance (MDP) and the mean nearest taxon distance (MNTD) described by Webb et al. (2002).

232 The phylogenetic distances measured in the observed communities were compared with those in 233 the null communities generated by randomization (1,000 permutations) by calculating 234 standardized effect sizes (SES). Tip labels were randomly shuffled across the tips of the 235 phylogeny for the null model. In this model, the community data matrix is not randomized in 236 order to fix most of the patterns (species richness, observed occupancy rates and OTU 237 abundance) and to allow the pattern of interest to vary (phylogenetic distance) (Swenson, 2014). 238 However, type I error rates may be inflated if a phylogenetic signal in OTU abundance occurs 239 (Hardy, 2008). As no phylogenetic signals in OTU abundance were found using Blomberg's $K$ 240 statistic (Blomberg et al., 2003) the use of this null model was validated. Negative SES values 241 and $p<0.05$ indicated phylogenetic clustering and smaller than expected phylogenetic distances 242 among co-occurring OTUs. Two analyses were performed to create the null communities, one 243 using all the OTUs and another one using the OTUs obtained separately from the DNA and the 244 RNA fractions. Both methods gave similar results and only results from the former analysis are 245 therefore presented.

\section{Phylogenetic signal}

Here we measured the 'mean expression ratio' of each OTU, in order to access the metabolic status of the microorganisms. This metric was obtained for each OTU by dividing the mean relative abundance in the RNA fractions (mean $R A_{R N A}$ ) by the sum of the mean relative abundance in the DNA (mean $R A_{D N A}$ ) and RNA fractions among all samples.

meanexpressionratio $=\frac{m e a n R A_{R N A}}{m_{\text {eanRA }} A_{R N A}+m e a n R A_{D N A}}$

with 
$254 \operatorname{meanRA}=\frac{\sum_{i=1}^{n} R A O T U i}{n}$

$255 n=$ number of plants showing the OTUi within their DNA and/or RNA fraction

256 The standard errors were then calculated for each OTU of the 19 samples. A zero value for this 257 ratio would indicate an absence of the OTU from the RNA fractions while a value of one would 258 indicate that the OTU was not detected in the DNA fractions. To determine whether the 'mean

259 260 expression ratio' across the phylogeny was not random, the phylogenetic signal was calculated using the Kse statistic (Blomberg et al., 2003; Ives et al., 2007) for the global phylogenetic tree and for phylogenies built for each main fungal phylum (i.e. Glomeromycota, Basidiomycota, and Ascomycota). This index integrates measurement errors of the trait by calculating the standard errors of the means for each OTU. The Kse statistic compares the observed phylogenetic signal in the 'mean expression ratio' to the signal obtained under a Brownian motion model of trait evolution. In this model, traits evolve as a random walk along the branch length of a phylogeny. The statistical significance of Kse was evaluated by comparing the observed patterns of variance of the independent contrasts of the trait to a null model. Taxa labels were shuffled across the tips of the phylogeny for the null model. A 'mean expression ratio' with $p<0.05$ indicated a nonrandom phylogenetic signal.

\section{Results}

DNA- and RNA-based analyses provided similar alpha and beta diversity profiles

\section{but different OTU richness levels}

We examined alpha and beta diversity measures of the fungal community colonizing the roots of 19 Agrostis stolonifera plants to see whether the nature of the co-extracted nucleic acids 
encapsulated different information. The mean Chao 1 index, that estimates 'true OTU diversity' by taking rare OTU into account, did not differ significantly $(p=0.11)$ between the DNA and RNA fractions $\left(\mathrm{S}\right.$. Chao $1_{\mathrm{DNA}}=215$, S.Chao $\left.1_{\mathrm{RNA}}=232\right)$. On average, the communities in the RNA fractions were not considered more diverse than those in the DNA fractions as not all of the Hill diversity numbers were significantly different (Fig. 1a). Nevertheless, sample-to-sample variations did exist (Fig. 1a). Interestingly, OTU richness, i.e. a special case of Hill diversity numbers when $\mathrm{q}=0$, was significantly higher in the RNA fractions than in the DNA fractions, with on average 149 OTUs in the RNA fractions and 133 OTUs in the DNA fractions $(p<0.01)$ (Fig. 1a). The sequencing depth was similar between both fractions (Fig. S1).The procrustes analyses indicated a significant similarity $(p<0.001)$ between the distances obtained from the DNA and RNA matrices. Thus, the beta diversity was not structured according to the type of nucleic acid.

0

Nucleic acid type impacts the perception of the taxonomic composition

The fungal communities in the roots were dominated by Pezizomycotina (Ascomycota) and Agaricomycotina (Basidiomycota) (Fig. S2). Each phylum, except for the Basidiomycota, was dominated by the same OTU in both the DNA and the RNA fractions, representing $17 \%$ to $76 \%$ of the total sequence number (Fig. 1b). The most abundant OTUs in the DNA and RNA fractions were the same except for the Basidiomycota. In this phylum, the two most abundant OTUs in the DNA and RNA fractions were assigned to the Agaricomycetes. The most abundant OTUs were assigned unambiguously to the class level (Sordariomycetes) within the Ascomycota, to the order level (Agaricales) within the Basidiomycota and to the family level (Glomeraceae) within the Glomeromycota (i.e. unknown at lower taxonomic rank). The mean relative sequence abundance assigned to the Basidiomycota was significantly higher in the DNA fraction than in 
302 the RNA fraction ( $p<0.05$, Fig. 1b). Conversely, sequences assigned to the Ascomycota were

303 more abundant in the RNA fraction than in the DNA fraction $(p<0.001$, Fig. 1b).

304 Glomeromycota represented $3.9 \%$ and $10.1 \%$ of the total number of sequences in the DNA and

305 RNA fractions respectively but the differences between these fractions were not significant (Fig. 306 1b).

\section{At the population level, very few fungal OTUs were shared by all co-occurring}

\section{plants}

Nine OTUs out of a total of 635 OTUs were found in both the DNA and RNA fractions of all of the 19 co-occurring $A$. stolonifera plants, accounting for $33 \%$ of the total number of sequences (Fig. 2). The number of sequences was equally divided into the RNA fraction (54\%) and the DNA fraction (46\%). Considering each fraction separately, 17 and 16 OTUs in the DNA and RNA fractions respectively were shared by all samples. All OTUs in the 'DNA core' were found in the RNA fraction of at least one sample and reciprocally. The number of OTUs shared by all samples in both fractions was negatively linked to the sampling size (Fig. S3). However, this number of shared OTUs stabilized at around 15 samples, indicating that the 'core microbiome' size would not be diminished by increased sampling within these co-occurring $A$. stolonifera (Fig. S3). The phylogenetic analysis (Fig. 2) revealed that the fungal 'core microbiome' was dominated by Ascomycota and the closest known sequence of the most abundant OTU was designated Ascomycete sp. (i.e. an unknown OTU) (Fig. 2). Strikingly, most of the OTUs in the fungal potential 'core microbiome' were only distantly related to known species (Fig. 2). 
325 We tested our working hypothesis that the host plant acts as an environmental filter on microbial

326 communities by examining the phylogenetic structure of the fungal root microbiome. For this we

327 measured the standardized effects size of the mean pairwise distance $\left(\mathrm{SES}_{\mathrm{MPD}}\right)$ and of the mean

328 nearest taxon distance ( $\mathrm{SES}_{\mathrm{MNTD}}$ ) in the DNA and RNA fractions of 19 samples (i.e. 38

329 fractions). Negative values of $\mathrm{SES}_{\mathrm{MPD}}$ indicated that the co-occurring fungal OTUs were more

330 closely related than expected under a null model, and this phylogenetic pattern was significant ( $p$

$331<0.05$ ) in 20 out of the 38 DNA and RNA fractions (Table 1). This phylogenetic clustering

332 based on the $\mathrm{SES}_{\mathrm{MPD}}$ measures was reinforced by the $\mathrm{SES}_{\mathrm{MNTD}}$ values (Table 1). The obtained

333 pattern was significant for 36 DNA and RNA fractions indicating that phylogenetic clustering

334 mainly concerned the leaves of the phylogeny (Table 1). The $\mathrm{SES}_{\mathrm{MNTD}}$ index calculates whether

335 the closest related OTUs (nearest taxon) tend to co-occur or not in the communities relatively to

336 the null distribution. Significant $\mathrm{SES}_{\mathrm{MNTD}}$ values and non-significant $\mathrm{SES}_{\mathrm{MPD}}$ values indicated

337 that the OTUs were phylogenetically structured near the tips of the phylogeny and randomly

338 distributed across the tree, i.e. deeper branches contributed less to the pattern.

\section{Phylogenetic signal}

The fungal root microbiome displayed phylogenetic clustering within each sample compared to the local pool of OTUs. We investigated whether this phylogenetic relatedness was linked to ecological similarity by searching for a phylogenetic signal, using the 'mean expression ratio' as a proxy for a functional trait. Sample-to-sample variations were taken into account in the Kse index. We found that our trait, i.e. the 'mean expression ratio', displayed a significant phylogenetic signal (Table 2). This was true for the global phylogeny including all the fungal OTUs and also for the phylogenies built for each fungal phylum (i.e. Glomeromycota (Fig. S4), Basidiomycota (Fig. 3), and Ascomycota (Fig. S5). Thus, the 'mean expression ratio' was linked 
349 to phylogenetic relatedness. The Kse statistic was less than one for all phylogenies except the

350 Basidiomycota phylogeny (Table 2). Kse values below one indicated that the OTUs were more

351 divergent in their 'mean expression ratio' than would be expected under a Brownian motion

352 model of trait evolution whereas a value above one indicated that the values of the 'mean

353 expression ratio' were more similar between phylogenetically related OTUs than between OTUs

354 drawn at random.

355

356

357

358

359

360

361

\section{Discussion}

In this study we assessed the complexity of the fungal root microbiome of co-occurring $A$. stolonifera and tested the hypothesis that fungal communities in plant roots are not random assemblages: we demonstrated for the first time that the phylogenetic signal of the fungal root microbiome was phylum dependent. This new understanding resulted from the application of DNA and RNA co-extraction strategy.

\section{OTU richness and taxonomic composition of the root fungal microbiome differed}

\section{between the DNA and RNA fractions}

In previous studies, the fungal community composition in roots was essentially analyzed either by cloning of PCR products (e.g. Vandenkoornhuyse et al., 2002a; 2002b) or more recently, by mass sequencing of amplicons using targeted DNA (e.g. Opik et al., 2012; Shakya et al., 2013a; Bonito et al., 2014). Others studies based on RNA and DNA extractions have used the RNA/DNA ratio as a proxy to investigate microbial dormancy and to estimate the metabolically active community(Aanderud et al., 2016, Jones and Lennon, 2010). In a similar way we defined 
373 the root fungal microbiome of co-occurring $A$. stolonifera plants by combining the DNA- and 374 RNA-based 18S rRNA amplicon analyses. However, the nucleic acids were co-extracted in order 375 to limit any extraction-related bias. We found higher OTU richness levels in the RNA fractions 376 than in the DNA fractions (Fig. 1a, $\mathrm{q}=0$ ), that was consistent with the study by Kuramae et al. (2013) and indicated that RNA-based approaches capture higher numbers of different OTUs in a given sample. At first glance this may seem surprising, but considering that RNA extracts contain roughly $80-90 \%$ of rRNA transcripts, the rRNA genes were much more diluted in the DNA fraction and could not be amplified. Thus, for a given quantity of nucleic acids, a higher abundance of targeted 18S rRNA genes was introduced into the RT-PCR mix possibly allowing the capture of more diverse molecules. Although not significantly different, the Chao 1 index exhibited the same trend of decreased richness in the DNA fraction. When other alpha diversity indexes were used, the differences were also not significant (Fig. 1a, q > 0.5). However, significant differences between the DNA and RNA-based analyses were detected when taxonomic composition was taken into account (Fig. 1b). The relative sequence abundance of the Basidiomycota was higher in the DNA fractions than in the RNA fractions and the opposite was true for the Ascomycota (Fig. 1b). It is tempting to conclude that the Basidiomycota was less 'active' than the Ascomycota phylum. However, while RNA represents expressed gene levels and is the best proxy for actively growing microorganisms at the nucleic acid level, there is not always a direct link between rRNA abundance, growth and activity in microorganisms (Blazewicz et al., 2013). The absence of a given OTU from the RNA-based analysis, but detected in the DNA, was not related to amplification bias (i.e. only OTUs found in the true replicates were kept). One possible explanation was that the OTUs detected from DNA fraction originated in part from dormant or dead cells. 
396 These results show that it is essential to be aware of the type of nucleic acid being used for

397 comparisons or meta-analyses and highlights the advantages and importance of taking both kinds of nucleic acids into account to gain deeper insights into microbial diversity.

\section{Fungal root microbiome is diverse and highly variable among co-occurring $A$.}

\section{stolonifera}

In the nineteen $A$. stolonifera plants, harvested from a natural ecosystem, we found 635 OTUs with a dominance of Pezizomycotina (Ascomycota) followed by Agaricomycotina (Basidiomycota). The Glomeromycota represented only a small proportion of the total number of OTUs (less than 4\%) in the DNA fraction, which is comparable to the fraction found in Agave species (Coleman-Derr et al., 2015). However, this proportion was higher in RNA fractions (10\%), underlining the interest of RNA based-analyses. A few published studies have focused on the fungal fraction of the root microbiome, mainly in woody plant species, by mass sequencing from DNA extracts (Gottel et al., 2011, Shakya et al., 2013a; Bonito et al., 2014; Coleman-Derr et al., 2015). Although these studies involved different primer pairs and different hosts, the fungal taxonomic composition at the subphylum level was similar to our study. The fungal 'core microbiome' at the $A$. stolonifera population level (i.e. core microbiome sensu Vandenkoornhuyse et al., 2015) accounted for $\sim 1.5 \%$ of the total OTUs (i.e. 9/635). The core microbiome is expected to decrease in complexity whereas the level of ecological organization will increase (i.e. from individual to ecosystem) (Vandenkoornhuyse et al., 2015). Although the level used to define the 'core microbiome' was low (i.e. a population of co-occurring plants) and no temporal changes were taken into account, the complexity of the fungal 'core microbiome' was lower than expected given the fact that all the $A$. stolonifera sampled in this study came from 
419 the same turf. Increased sampling within this $A$. stolonifera population would be unlikely to 420 modify the number of shared OTUs (Fig. S3), but might increase the total number of OTUs

detected. Moreover, the OTUs shared by all the plants in both their DNA and RNA fractions were mainly abundant OTUs affiliated to Pezizomycotina (Ascomycota), which represented more than $30 \%$ of all the sequences.

An increased sequencing depth would lead to an increase of the rare, rather than abundant, OTUs. Unexpectedly, only 1 OTU affiliated to the Glomeromycota forming arbuscular mycorrhiza with $A$. stolonifera was shared by all individuals, while most of the other OTUs shared by all individuals were unknown Ascomycota forming unknown fungal endophytes (Fig. 2). As the different Ascomycota and Basidiomycota fungal endophytes are acknowledged to be involved in plant resistance to stresses (e.g. Soares et al., 2016; Cosme et al., 2016), our findings raise important new questions about the functions of these unknown endophytes and more widely emphasize the need for a more holistic perception and understanding of the plant holobiont (Vandenkoornhuyse et al., 2015).

\section{A. stolonifera fungal endophytic communities exhibited significant phylogenetic clustering}

We found that the fungal communities in plant roots exhibited a significant phylogenetic structure whether the analyses were based on DNA or RNA which confirmed our working hypothesis that the fungal communities in plant roots are not random assemblages (Table 1). At the host-plant scale, the phylogenetic structure of the fungal microbiome showed greater clustering than would be expected under a null model. Thus, the fungal OTUs in an individual plant were more closely related to each other than to the pool detected at the community level. It is not easy to discriminate between the different neutral or ecological processes underlying this pattern because various explanations are feasible (Losos, 2008, Revell et al., 2008). Indeed, 
443 Phylogenetic clustering can be generated by dispersal limitation as well as by inter-specific 444 interactions or environmental filtering (Bell, 2005; Cavender-Bares et al., 2009; Helmus et al., 2007). However, the spatial scale of our study was small in relation to the known dispersal capacities of fungi (Taylor et al., 2006). Dispersal limitation was therefore unlikely or, at least, would not be the main driver of the observed phylogenetic pattern. Ectomycorrhizal fungal communities in plant roots have been shown to be shaped by competitive interactions (Pickles $e t$ al., 2012) and host-plant specificity or preference has also been reported (Ishida et al., 2007, Tedersoo et al., 2013). Similarly, assembly of the arbuscular mycorrhizal fungal community in plant roots reflects a degree of host-plant preferences (Vandenkoornhuyse et al., 2002b, 2003;

Davison et al., 2012). In Populus deltoides roots, the endophytic fungal communities were found to be clearly distinct from the surrounding rhizospheric communities (Gottel et al., 2011; Shakya et al., 2013a). Environmental filtering by the host, which would represent a specific habitat, could thus be an important mechanism leading to phylogenetic clustering. Assuming that phylogenetically close OTUs share common phenotypic traits (Webb et al., 2002), the host plant would select for OTUs with particular biological features, adapted to a symbiotic life style. This implies that traits favouring symbiosis would be phylogenetically conserved in fungi. Environmental filtering is probably not an exclusive mechanism and other processes, such as inter-specific microbial interactions, might explain the observed phylogenetic structure. For instance, if competitive ability is assumed to be correlated with phylogenetic distance then competition can drive phylogenetic clustering (Mayfield \& Levine, 2010). Mechanisms leading to an over-dispersed phylogenetic structure, especially inter-species competition, which classically leads to species exclusion between relatives (Diamond 1975), would have a weaker effect than environmental filtering by the host. Kin selection, a strategy favouring the 
reproductive success of an organism's relatives, might also be important in structuring fungal communities.

\section{Disentangling the processes underlying the observed phylogenetic structure}

The relative importance of the above-mentioned processes in explaining the observed phylogenetic clustering can be elucidated by detecting the phylogenetic signal of relevant trait(s) (Mayfield \& Levine, 2010). However, phenotypic traits are especially difficult to measure in complex communities, particularly for uncultivated microbes. In this study, we used the 'mean expression ratio' to access the microbial metabolic status. Sample-to-sample variations were taken into account using the Kse index that allowed the power of the test to be increased and avoided an estimation bias (Hardy \& Pavoine, 2012). Interestingly, a significant phylogenetic signal was found for all fungal groups (Table 2). Within the Ascomycota and Glomeromycota, the 'mean expression ratios' of evolutionary-related OTUs were more divergent than would be expected under a Brownian motion model of trait evolution (Table 2, Fig. S4, S5). Conversely, the OTUs in the Basidiomycota were more similar than expected, i.e. related OTUs shared similar expression ratios (Table 2, Fig. 3). Thus, the combined information about the phylogenetic structure (Table 1) and the phylogenetic signals analyses (Table 2) suggested that the Basidiomycota assemblage was mainly governed by environmental filtering, favouring the co-existence of related and similar OTUs in their 'mean expression ratio'. In contrast, the Ascomycota and Glomeromycota assemblages were more impacted by competitive interactions promoting the co-existence of phylogenetically related but dissimilar OTUs in their 'mean expression ratio'. In this latter case, competitive ability would be positively correlated with phylogenetic distance or, competition would drive character displacement rather than OTUs exclusion. Other relevant functional traits now need to be used to confirm our interpretations and 
490 to improve our detection and understanding of the phylogenetic signal. One possibility would be

491 to perform a comparative transcriptomic analysis of the fungal root microbiome, although a

492 number of methodological padlocks would first need to be broken, notably the selective

493 extraction of fungal RNA.

494

495

496

497

498

499

500

\section{Conclusion and prospects}

We describe here the root fungal microbiome associated with an $A$. stolonifera population using a molecular strategy combining DNA- and RNA-based approaches. We were able for the first time to draw up a comprehensive picture of the phylogenetic patterns existing in the fungal root microbiome by examining the phylogenetic structure and measuring the phylogenetic signal. We found that the fungal communities were not randomly assembled but instead appeared to be specifically filtered by their plant host. We thus provide new insights into the rules of assembly governing the root fungal microbiome community. The limited number of OTUs shared by all individuals and the clustered phylogenetic structure suggested that each plant recruits a particular microbial community to adapt to environmental conditions at a microscale.

The use of evolutionary information to describe an ecological pattern is a first step towards a full understanding of community assembly. Further experimental studies are now needed to focus on the processes underlying these patterns.

\section{Acknowledgments}

We are very grateful to Diana Warwick and the four anonymous reviewers for comments and suggested modifications on a previous version of the manuscript, Biogenouest Genomics and the Human and Environmental Genomics platform (https://geh.univ-rennes1.fr/) for the sequencing, and the Genouest Bioinformatics facilities. 


\section{References}

Aanderud ZT, Vert JC, Lennon JT, Magnusson TW, Breakwell DP, Harker AR. 2016. Bacterial Dormancy Is More Prevalent in Freshwater than Hypersaline Lakes. Frontiers in Microbiology 7: Article 853.

Bell G. 2005. The co-distribution of species in relation to the neutral theory of community ecology. Ecology 86: 1757-1770.

Ben Maamar S, Aquilina L, Quaiser A, Pauwels H, Michon-Coudouel S, Vergnaud-Ayraud V, Labasque T, Roques C, Abbott BW, Dufresne A. 2015. Groundwater Isolation Governs Chemistry and Microbial Community Structure along Hydrologic Flowpaths. Frontiers in Microbiology 6: Article 1457.

Blazewicz SJ, Barnard RL, Daly RA, Firestone MK. 2013. Evaluating rRNA as an indicator of microbial activity in environmental communities: limitations and uses. ISME Journal 7: 2061-2068.

Blomberg SP, Garland T, Ives AR. 2003. Testing for phylogenetic signal in comparative data: behavioral traits are more labile. Evolution 57: 717-745.

Bonito G, Reynolds H, Robeson MS II, Nelson J, Hodkinson BP, Tuskan G, Schadt CW, Vilgalys R. 2014. Plant host and soil origin influence fungal and bacterial assemblages in the roots of woody plants. Molecular Ecology 23: 3356-3370.

Borneman J, Hartin RJ. 2000. PCR primers that amplify fungal rRNA genes from environmental samples. Applied and Environmental Microbiology 66: 4356-4360.

Bulgarelli D, Rott M, Schlaeppi K, Ver Loren van Themaat E, Ahmadinejad N, Assenza F, Rauf P, Huettel B, Reinhardt R, Schmelzer E, Peplies J, Gloeckner FO, Amann R, Eickhorst T, Schulze-Lefert P. 2012. Revealing structure and assembly cues for Arabidopsis root-inhabiting bacterial microbiota. Nature 488: 91-95.

Cahill JF Jr., Kembel SW, Lamb EG, Keddy PA. 2008. Does phylogenetic relatedness influence the strength of competition among vascular plants? Perspectives in Plant Ecology, 
Evolution and Systematics 10: 41-50.

Cavender-Bares J, Kozak KH, Fine PVA, Kembel SW. 2009. The merging of community ecology and phylogenetic biology. Ecology Letters 12: 693-715.

Chao A. 1984. Nonparametric estimation of the number of classes in a population. Scandinavian Journal of Statistics 11:265-270.

Ciobanu M-C, Burgaud G, Dufresne A, Breuker A, Rédou V, Ben Maamar S, Gaboyer F, Vandenabeele-Trambouze O, Lipp JS, Schippers A, Vandenkoornhuyse P, Barbier G, Jebbar M, Godfroy A, Alain K. 2014. Microorganisms persist at record depths in the subseafloor of the Canterbury Basin. ISME Journal 8: 1370-1380.

\section{Coleman-Derr D, Desgarennes D, Fonseca-García C, Gross S, Clingenpeel S, Woyke T,} North G, Visel A, Partida-Martínez LP, Tringe SG. 2015. Plant compartment and biogeography affect microbiome composition in cultivated and native Agave species. New Phytologist 209: 798-811.

Cosme M, Lu J, Erb M, Stout MJ, Franken P, Wurst S. 2016. A fungal endophyte helps plants to tolerate root herbivory through changes in gibberellin and jasmonate signaling. New Phytologist 211: 1065-1076.

Darriba D, Taboada GL, Doallo R, Posada D. 2012. jModelTest 2: more models, new heuristics and parallel computing. Nature Methods 9: 772.

Davison J, ÖPIK M, Zobel M, Vasar M, Metsis M, Moora M. 2012. Communities of arbuscular mycorrhizal fungi detected in forest soil are spatially heterogeneous but do not vary throughout the growing season. PLOS ONE 7: e41938.

Diamond JM. 1975. Assembly of species communities. In: Cody ML, Diamond JM eds. Ecology and evolution of communities. Cambridge, Massachusetts, USA: Harvard University Press, 342-444.

Diaz S, Cabido M. 2001. Vive la difference: plant functional diversity matters to ecosystem processes. Trends in Ecology \& Evolution 16: 646-655. 
Fonseca-García C, Coleman-Derr D, Garrido E, Visel A, Tringe SG, Partida-Martínez LP. 2016. The cacti microbiome: interplay between habitat-filtering and host-specificity. Frontiers in Microbiology 7: Article 891.

\section{Gottel NR, Castro HF, Kerley M, Yang Z, Pelletier DA, Podar M, Karpinets T,} Uberbacher E, Tuskan GA, Vilgalys R, Doktycz M, Schadt CW. 2011. Distinct microbial communities within the endosphere and rhizosphere of Populus deltoides roots across contrasting soil types. Applied and Environmental Microbiology 77: 5934-5944.

Jones S, Lennon J. 2010. Dormancy contributes to the maintenance of microbial diversity. Proceedings of the National Academy of Sciences of the USA 107: 5881-5886.

Hardy OJ. 2008. Testing the spatial phylogenetic structure of local communities: statistical performances of different null models and test statistics on a locally neutral community. Journal of Ecology 96: 914-926.

Hardy OJ, Pavoine S. 2012. Assessing phylogenetic signal with measurement error: a comparison of Mantel tests, Blomberg et al.'s K, and phylogenetic distograms. Evolution 66: 2614-2621.

Helmus MR, Savage K, Diebel MW, Maxted JT, Ives AR. 2007. Separating the determinants of phylogenetic community structure. Ecology Letters 10: 917-925.

Hill M. 1973. Diversity and evenness: a unifying notation and its consequences. Ecology54: $427-432$.

Ishida TA, Nara K, Hogetsu T. 2007. Host effects on ectomycorrhizal fungal communities: insight from eight host species in mixed conifer-broadleaf forests. New Phytologist 174: 430440.

Ives AR, Midford PE, Garland TJR. 2007. Within-Species Variation and Measurement Error in Phylogenetic Comparative Methods. Systematic Biology 56: 252-270.

Kembel SW. 2009. Disentangling niche and neutral influences on community assembly: assessing the performance of community phylogenetic structure tests. Ecology Letters 12: 949- 
960.

Kembel SW, Cowan PD, Helmus MR, Cornwell WK, Morlon H, Ackerly DD, Blomberg SP, Webb CO. 2010. Picante: R tools for integrating phylogenies and ecology. Bioinformatics 26: $1463-1464$.

Klein A, Bohannan B, Jaffe D, Levin D, Green J. 2016. Molecular Evidence for Metabolically Active Bacteria in the Atmosphere. Frontiers in Microbiology 7: Article 772.

Kuramae EE, Verbruggen E, Hillekens R, de Hollander M, Röling WFM, van der Heijden MGA, Kowalchuk GA. 2013. Tracking fungal community responses to maize plants by DNAand RNA-based pyrosequencing. PLoS ONE 8: e69973.

Lambais MR, Crowley DE, Cury JC, Büll RC, Rodrigues RR. 2006. Bacterial diversity in tree canopies of the Atlantic forest. Science 312: 1917.

Losos JB. 2008. Phylogenetic niche conservatism, phylogenetic signal and the relationship between phylogenetic relatedness and ecological similarity among species. Ecology Letters 11: 995-1003.

Ludwig W, Strunk O, Westram R, Richter L, Meier H, Yadhukumar, Buchner A, Lai T, Steppi S, Jobb G, Förster W, Brettske I, Gerber S, Ginhart AW, Gross O, Grumann S, Hermann S, Jost R, König A, Liss T, Lüßmann R, May M, Nonhoff B, Reichel B, Strehlow R, Stamatakis A, Stuckmann N, Vilbig A, Lenke M, Ludwig T, Bode A, Heinz Schleifer K 2004. ARB: a software environment for sequence data. Nucleic Acids Research 32: $1363-1371$.

Lundberg DS, Lebeis SL, Paredes SH, Yourstone S, Gehring J, Malfatti S, Tremblay J, Engelbrektson A, Kunin V, del Rio TG, Edgar RC, Eickhorst T, Ley RE, Hugenholtz P, Green Tringe S, Dangl JL 2012. Defining the core Arabidopsis thaliana root microbiome. Nature 488: 86-90.

Martiny AC, Treseder K, Pusch G. 2013. Phylogenetic conservatism of functional traits in microorganisms. ISME Journal 7: 830-838. 
Mayfield MM, Levine JM. 2010. Opposing effects of competitive exclusion on the phylogenetic structure of communities. Ecology Letters 13: 1085-1093.

Nunes F, Aquilina L, de Ridder J, Francez A, Quaiser A, Caudal J, Vandenkoornhuyse P, Dufresne A. 2015. Time-scales of hydrological forcing on the geochemistry and bacterial community structure of temperate peat soils. Scientific Reports 5: 14612.

Oksanen J, Blanchet G, Kindt R, Minchin PR, Legendre P, O'Hara B, Simpson LG, Solymos P, Stevens M, Wagner H. 2011. Vegan: Community Ecology Package. R package Version 2.0-2. cran.r-project.org.

Opik M, Zobel M, Cantero J, Davison J, Facelli J, Hiiesalu I, Jairus T, Kalwij J, Koorem K, Leal M, Liira J, Metsis M, Neshataeva V, Paal J, Phosri C, Polme S, Reier Ü, Saks Ü, Schimann H, Thiéry O, Vasar M, Moora M. 2013. Global sampling of plant roots expands the described molecular diversity of arbuscular mycorrhizal fungi. Mycorrhiza 23: 411-430.

Peiffer JA, Spor A, Koren O, Jin Z, Tringe SG, Dangl JL, Buckler ES, Ley RE. 2013. Diversity and heritability of the maize rhizosphere microbiome under field conditions. Proceedings of the National Academy of Sciences of the USA 110: 6548-6553.

Peres-Neto PR, Jackson DA. 2001. How well do multivariate data sets match? The advantages of a Procrustean superimposition approach over the Mantel test. Oecologia 129: 169-178.

Pickles BJ, Genney DR, Anderson IC, Alexander IJ. 2012. Spatial analysis of ectomycorrhizal fungi reveals that root tip communities are structured by competitive interactions. Molecular Ecology 21: 5110-5123.

Pruesse E, Peplies J, Glockner FO. 2012. SINA: Accurate high-throughput multiple sequence alignment of ribosomal RNA genes. Bioinformatics 28: 1823-1829.

Quast C, Pruesse E, Yilmaz P, Gerken J, Schweer T, Yarza P, Peplies J, Glöckner FO. 2013. The SILVA ribosomal RNA gene database project: improved data processing and webbased tools. Nucleic Acids Research 41: D590-6.

R Development Core Team. 2013. R: A language and environment for statistical computing, $R$ 
Foundation for Statistical Computing, Vienna, Austria. Version 3.0.2. www.R-project.org.

Revell L, Harmon L, Collar D. 2008. Phylogenetic Signal, Evolutionary Process, and Rate. Systematic Biology 57: 591-601.

Rice P, Longden I, Bleasby A. 2000. EMBOSS: the European Molecular Biology Open Software Suite. Trends in Genetics 16: 276-277.

Ronquist F, Teslenko M, van der Mark P, Ayres DL, Darling A, Höhna S, Larget B, Liu L, Suchard MA, Huelsenbeck JP. 2012. MrBayes 3.2: efficient Bayesian phylogenetic inference and model choice across a large model space. Systematic Biology 61: 539-542.

Schlaeppi K, Dombrowski N, Oter RG, van Themaat EVL, Schulze-Lefert P. 2014.

Quantitative divergence of the bacterial root microbiota in Arabidopsis thaliana relatives. Proceedings of the National Academy of Sciences of the USA 111: 585-592.

Shakya M, Gottel N, Castro H, Yang ZK, Gunter L, Labbé J, Muchero W, Bonito G, Vilgalys R, Tuskan G, Podar M, Schadt CW. 2013a. A multifactor analysis of fungal and bacterial community structure in the root microbiome of mature Populus deltoides trees. PLoS ONE 8: e76382.

Shakya M, Quince C, Campbell JH, Yang ZK, Schadt CW, Podar M. 2013b. Comparative metagenomic and rRNA microbial diversity characterization using archaeal and bacterial synthetic communities. Environmental Microbiology 15: 1882-1899.

Soares MA, Li HY, Kowalski KP, Bergen M, Torres MS, White JF. 2016. Evaluation of the functional roles of fungal endophytes of Phragmites australis from high saline and low saline habitats. Biological Invasions 18: 2689-2702.

Swenson N. 2014. Functional and phylogenetic ecology in R. Springer Science \& Business Media.

Taylor JW, Turner E, Townsend JP, Dettman JR, Jacobson D. 2006. Eukaryotic microbes, species recognition and the geographic limits of species: examples from the kingdom Fungi. Philosophical Transactions of the Royal Society B: Biological Sciences 361: 1947-1963. 
672 Tedersoo L, Mett M, Ishida TA, Bahram M. 2013. Phylogenetic relationships among host 673 plants explain differences in fungal species richness and community composition in ectomycorrhizal symbiosis. New phytologist 199: 822-831.

675 676

677

Valiente-Banuet A, Verdú M. 2007. Facilitation can increase the phylogenetic diversity of plant communities. Ecology Letters 10: 1029-1036.

Vandenkoornhuyse P, Baldauf SL, Leyval C, Straczek J, Young JPW. 2002a. Extensive fungal diversity in plant roots. Science 295: 2051.

Vandenkoornhuyse P, Husband R, Daniell TJ, Watson IJ, Duck JM, Fitter AH, Young JPW. 2002b. Arbuscular mycorrhizal community composition associated with two plant species in a grassland ecosystem. Molecular Ecology 11: 1555-1564.

Vandenkoornhuyse P, Ridgway KP, Watson IJ, Fitter AH, Young JPW. 2003. Co-existing grass species have distinctive arbuscular mycorrhizal communities. Molecular Ecology 12: 3085-3095.

Vandenkoornhuyse P, Mahé S, Ineson P, Staddon P, Ostle N, Cliquet J-B, Francez A-J, Fitter AH, Young JPW. 2007. Active root-inhabiting microbes identified by rapid incorporation of plant-derived carbon into RNA. Proceedings of the National Academy of Sciences of the USA 104: 16970-16975.

Vandenkoornhuyse P, Quaiser A, Duhamel M, Lê Van A, Dufresne A. 2015. The importance of the microbiome of the plant holobiont. New phytologist 206: 1196-1206.

Webb CO, Ackerly DD, McPeek MA, Donoghue MJ. 2002. Phylogenies and Community Ecology. Annual Review of Ecology and Systematics 33: 475-505. 
695

696

697

698

699

700

701

702

703

704

705

706

707

708

709

710

711

712

713

714

715

716

717

718

\section{Figure legends:}

Figure 1. Alpha diversity and taxonomic composition of fungal communities in DNA and RNA fractions.(a) Distribution of Hill diversity numbers in DNA and RNA sequence data analyses.

Different commonly used diversity indexes are special cases of Hill numbers (e.g. $q=0$

corresponds to the species richness $\mathrm{S}, \mathrm{q}=1$ corresponds to the exponential of the Shannon-

Wiener diversity index, $\mathrm{q}=2$ corresponds to the inverse Simpson index). Asterisks indicate significantly different means between DNA and RNA fractions (paired t-tests). 'ns': $p>0.05,{ }^{\prime}$ ': $p<0.05,{ }^{* * '}: p<0.01$. (b) Taxonomic composition of fungal communities in DNA and RNA fractions. Assignation of sequence taxonomy using SILVA database 115. Average relative sequence abundance $(\% \pm \mathrm{se})$ of each phylum in the fungal kingdom for 19 samples. Asterisks indicate significantly different means between DNA and RNA fractions. 'ns': $p>0.05,{ }^{\prime *}: p<$ $0.05,{ }^{\prime * * * 1}: p<0.001$. The mean relative sequence abundances of the two most abundant OTUs are shown for each phylum. The most abundant OTUs in the DNA and RNA fractions were the same except for Basidiomycota. For this latter group, the second most abundant OTU in the RNA fraction was the most abundant OTU in the DNA fraction. There was just a slight difference in sequence number between the first and second most abundant OTU in the RNA fraction (420 and 322 sequences respectively) and these two OTUs were assigned to the Agaricomycetes class.

Figure 2. Phylogenetic tree of the core microbiome OTUs at the level of 19 co-occurring Agrostis stolonifera plants. Tree construction was based on maximum likelihood. Only bootstrap values above 50 are indicated. The tree was constructed using sequences representative of the OTUs (taxa without names) and the closest reference sequences (taxa names in italic) from the 
719 non-redundant SILVA SSURef ARB database (release 115). '*': taxa belonging to the 'DNA

720 core', ' $\uparrow$ ': taxa belonging to the 'RNA core'. Stacked bars indicate the mean relative abundance

721 of each taxon in the DNA (blue) and RNA (red) fractions of the 19 samples. Some taxa

722 belonging to the 'DNA core' are also found in the RNA fractions but not in all samples and 723 reciprocally.

Figure 3. Phylogenetic tree of the Basidiomycota related root fungal microbiome OTUs. ML tree based on 432 bp of SSU rRNA gene sequences amplified from roots of Agrostis stolonifera. The tree was constructed using representative sequences of the OTUs (taxa without names) and the closest reference sequences (taxa names in italic) from the non-redundant SILVA SSURef ARB database (release 115). Barplots represent the mean expression ratio for each OTU among all samples. Null values indicate that this OTU was not detected in the RNA fraction, value $=1$ indicates that this OTU was not detected in the DNA fraction, value $=0.5$ indicates that the sum of the relative abundance between DNA and RNA fractions was equal. Green bars: values below 0.5 , red bars: values $\geq 0.5$. Error bars indicate $\pm S E$. Grey circles indicate the relative abundance of each OTU in the whole dataset. Node support values above 50 are given in the following order: bootstrap values and Bayesian posterior probabilities. 


\section{Figure 1}

Figure 1. Alpha diversity and taxonomic composition of fungal communities in DNA and RNA fractions.

(a) Distribution of Hill diversity numbers in DNA and RNA sequence data analyses. Different commonly used diversity indexes are special cases of Hill numbers (e.g. $q=0$ corresponds to the species richness $S, q=1$ corresponds to the exponential of the Shannon-Wiener diversity index, $q=2$ corresponds to the inverse Simpson index). Asterisks indicate significantly different means between DNA and RNA fractions (paired t-tests). 'ns': $p>0.05,{ }^{\prime} * 1: p<0.05$, '**': $p<0.01$. (b) Taxonomic composition of fungal communities in DNA and RNA fractions. Assignation of sequence taxonomy using SILVA database 115. Average relative sequence abundance ( $\% \pm$ se) of each phylum in the fungal kingdom for 19 samples. Asterisks indicate significantly different means between DNA and RNA fractions. 'ns': $p>0.05,{ }^{2 * 1}: p<0.05,{ }^{\prime * * * 1}$ : $p<0.001$. The mean relative sequence abundances of the two most abundant OTUs are shown for each phylum. The most abundant OTUs in the DNA and RNA fractions were the same except for Basidiomycota. For this latter group, the second most abundant OTU in the RNA fraction was the most abundant OTU in the DNA fraction. There was just a slight difference in sequence number between the first and second most abundant OTU in the RNA fraction (420 and 322 sequences respectively) and these two OTUs were assigned to the Agaricomycetes class. 

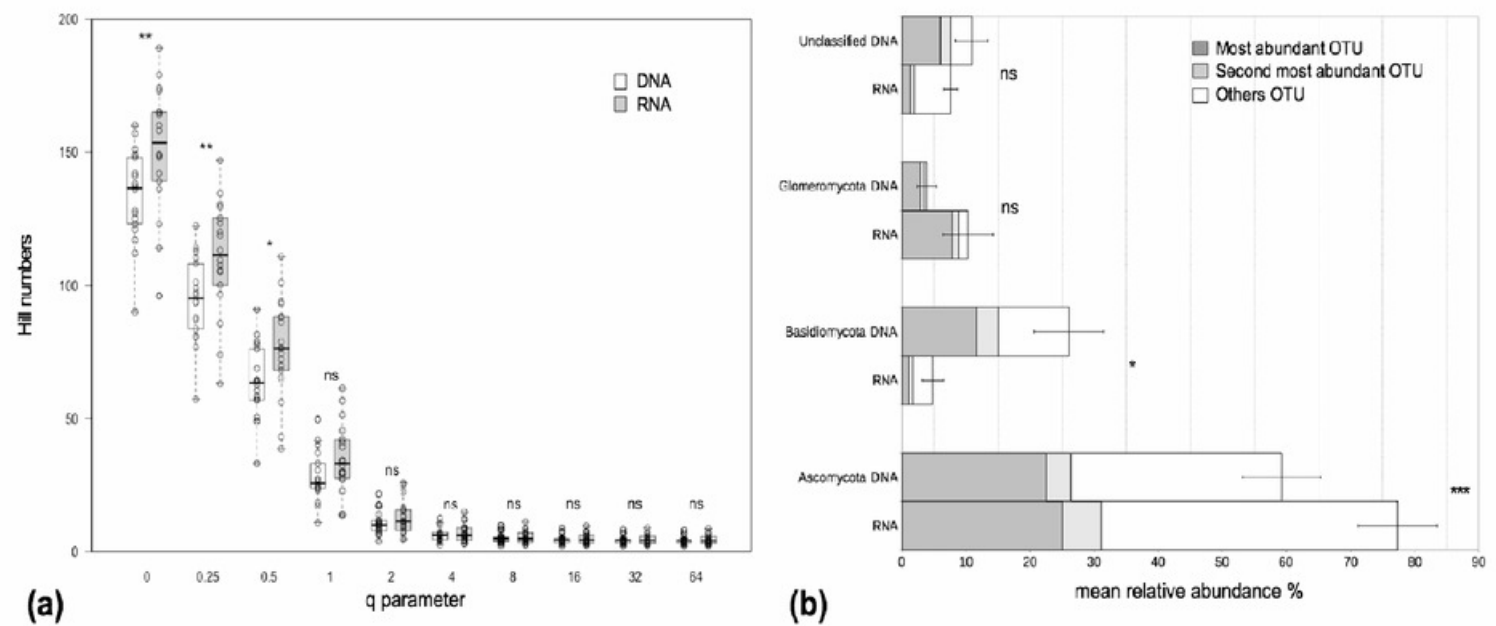


\section{Figure 2}

Figure 2. Phylogenetic tree of the core microbiome OTUs.

Phylogenetic tree of the core microbiome OTUs at the level of 19 co-occurring Agrostis stolonifera plants. Tree construction was based on maximum likelihood. Only bootstrap values above 50 are indicated. The tree was constructed using sequences representative of the OTUs (taxa without names) and the closest reference sequences (taxa names in italic) from the non-redundant SILVA SSURef ARB database (release 115). '*': taxa belonging to the 'DNA core', ' + ': taxa belonging to the 'RNA core'. Stacked bars indicate the mean relative abundance of each taxon in the DNA (blue) and RNA (red) fractions of the 19 samples. Some taxa belonging to the 'DNA core' are also found in the RNA fractions but not in all samples and reciprocally. 


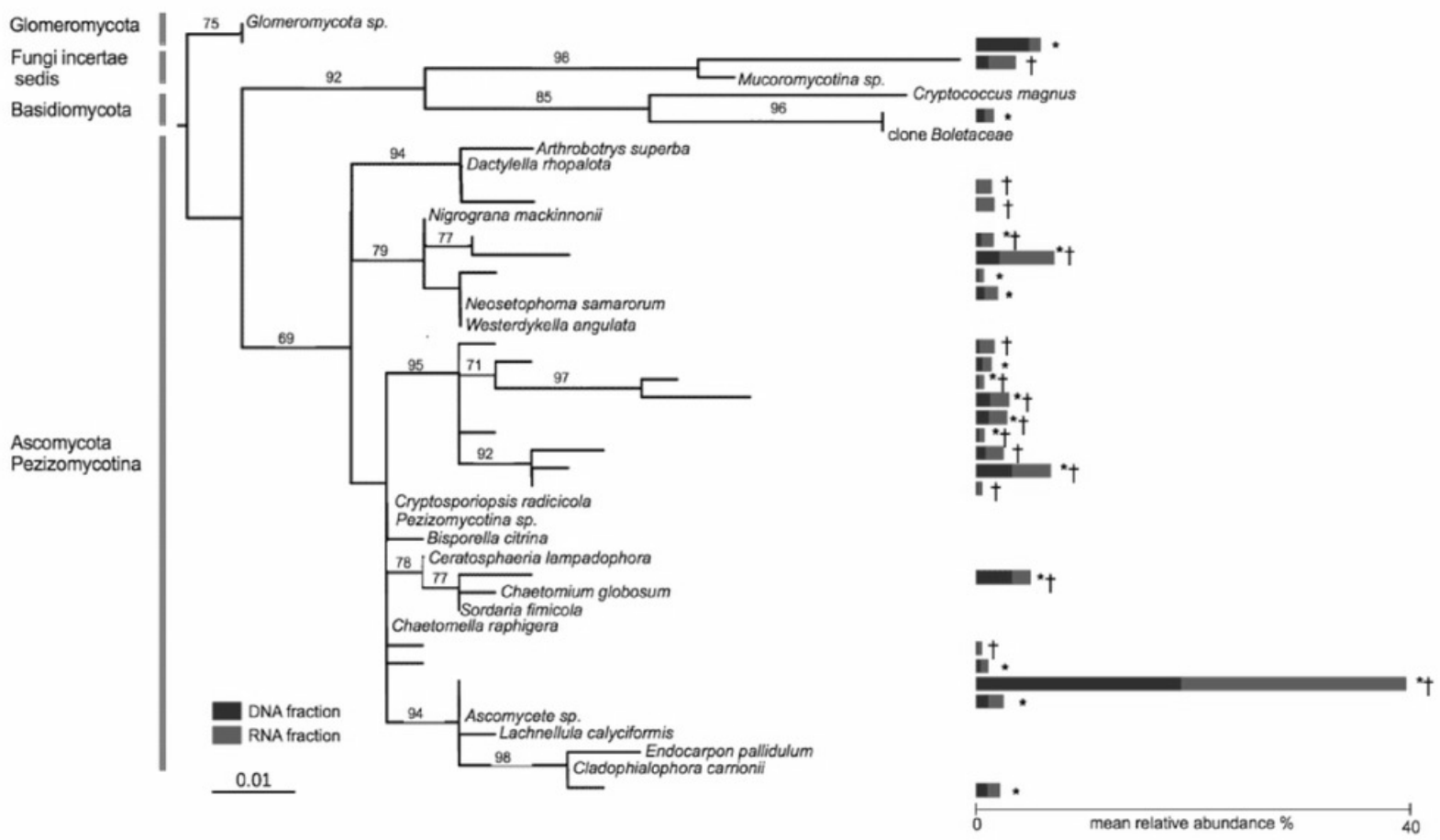




\section{Figure 3}

Figure 3. Phylogenetic tree of the Basidiomycota

Phylogenetic tree of the Basidiomycota related root fungal microbiome OTUs. ML tree based on 432 bp of SSU rRNA gene sequences amplified from roots of Agrostis stolonifera. The tree was constructed using representative sequences of the OTUs (taxa without names) and the closest reference sequences (taxa names in italic) from the non-redundant SILVA SSURef ARB database (release 115). Barplots represent the mean expression ratio for each OTU among all samples. Null values indicate that this OTU was not detected in the RNA fraction, value = 1 indicates that this OTU was not detected in the DNA fraction, value $=0.5$ indicates that the sum of the relative abundance between DNA and RNA fractions was equal. Green bars: values below 0.5 , red bars: values $\geq 0.5$. Error bars indicate $\pm \mathrm{SE}$. Grey circles indicate the relative abundance of each OTU in the whole dataset. Node support values above 50 are given in the following order: bootstrap values and Bayesian posterior probabilities. 

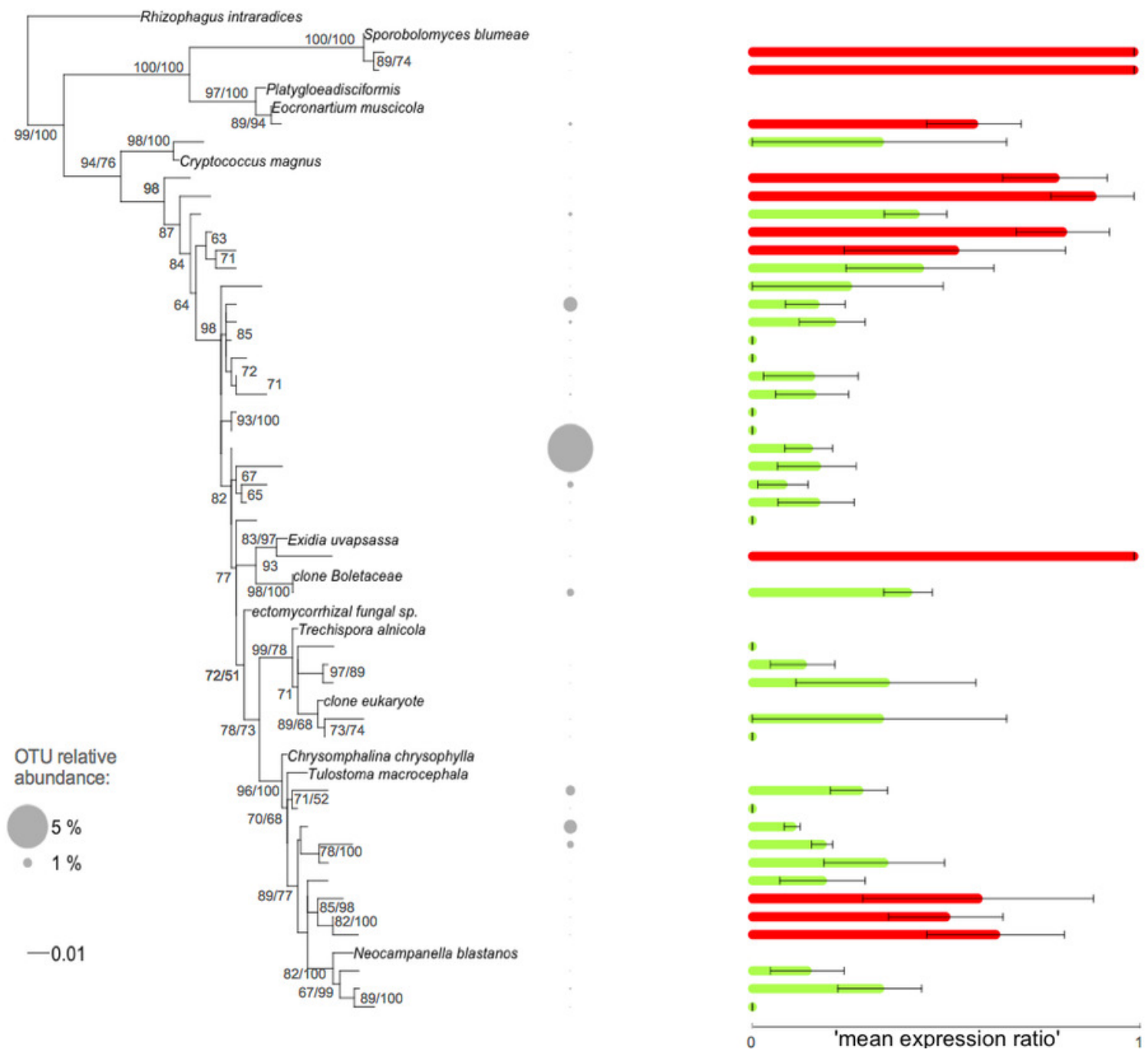


\section{Table $\mathbf{1}$ (on next page)}

Table 1. Phylogenetic clustering : SES index

Standardized effect sizes of the mean pairwise distance values $\left(\mathrm{SES}_{\mathrm{MPD}}\right)$ and standardized effect sizes of the mean nearest taxon distance values $\left(\mathrm{SES}_{\mathrm{MNTD}}\right)$ for the 19 fungal communities in Agrostis stolonifera roots detected in the DNA and RNA fractions. 
1 Table 1. Standardized effect sizes of the mean pairwise distance values $\left(\mathrm{SES}_{\mathrm{MPD}}\right)$ and

2 standardized effect sizes of the mean nearest taxon distance values (SES $\mathrm{SNND}_{\mathrm{M}}$ ) for the 19 fungal

3 communities in Agrostis stolonifera roots detected in the DNA and RNA fractions.

4

5

\begin{tabular}{cccccc}
\hline & nb OTU & \multicolumn{2}{c}{ SES $_{\text {MPD }}$} & \multicolumn{2}{c}{ SES } \\
Mamp \\
\cline { 2 - 6 } SaNA & DNA RNA & DNA & RNA & DNA & RNA \\
\hline 1 & $137 / 121$ & -0.14 & 1.27 & $-3.45^{*}$ & $-3.48^{*}$ \\
2 & $170 / 185$ & $-2.85^{*}$ & -0.18 & $-3.92^{*}$ & $-1.96^{*}$ \\
3 & $98 / 137$ & $-4.78^{*}$ & $-6.31^{*}$ & $-2.35^{*}$ & $-4.1^{*}$ \\
4 & $113 / 176$ & -1.09 & -1.65 & $-3.27^{*}$ & $-2.15^{*}$ \\
5 & $132 / 180$ & -0.58 & $-2.13^{*}$ & $-3.23^{*}$ & 0.02 \\
6 & $144 / 180$ & -1.09 & $-2.77^{*}$ & $-1.87^{*}$ & $-2.77^{*}$ \\
7 & $93 / 145$ & $-3.42^{*}$ & $-3.53^{*}$ & $-2.82^{*}$ & -1.53 \\
8 & $111 / 100$ & $-6.02^{*}$ & $-3.97^{*}$ & $-4.2^{*}$ & $-2.2^{*}$ \\
9 & $87 / 160$ & -1.51 & $-1.76^{*}$ & $-3.71^{*}$ & $-3.85^{*}$ \\
10 & $124 / 102$ & $-2.45^{*}$ & 0.78 & $-2.61^{*}$ & $-2.95^{*}$ \\
11 & $157 / 175$ & 0.31 & -0.18 & $-1.72^{*}$ & $-2.02^{*}$ \\
12 & $93 / 197$ & -0.45 & -1.05 & $-3.9^{*}$ & $-2.3^{*}$ \\
13 & $87 / 165$ & $-1.7^{*}$ & $-1.81^{*}$ & $-3.71^{*}$ & $-2.26^{*}$ \\
14 & $123 / 155$ & -1.36 & 0.04 & $-3.45^{*}$ & $-2.74^{*}$ \\
15 & $154 / 164$ & -1.51 & -1.55 & $-3.96^{*}$ & $-3.23^{*}$ \\
16 & $138 / 126$ & $-2.14^{*}$ & $-1.83^{*}$ & $-4.46^{*}$ & $-4.13^{*}$ \\
17 & $88 / 142$ & -1.35 & $-2.13^{*}$ & $-2.82^{*}$ & $-2.46^{*}$ \\
18 & $152 / 104$ & $-2.64^{*}$ & $-2.38^{*}$ & $-3.81^{*}$ & $-2.16^{*}$ \\
19 & $157 / 113$ & $-2.59^{*}$ & $-2.83^{*}$ & $-4.33^{*}$ & $-3.26^{*}$ \\
\hline
\end{tabular}

nb OTU: number of OTU in the community excluded reference species

*Community significantly structured $(\mathrm{P}<0.05)$

6

7 


\section{Table 2 (on next page)}

Table 2. Phylogenetic signal

Phylogenetic signal in phylogenies. 
1 Table 2. Phylogenetic signal in phylogenies.

2

\begin{tabular}{cc}
\hline Phylum & Kse \\
\hline Ascomycota & $0.41 * *$ \\
Basidiomycota & $1.08 * * *$ \\
Glomeromycota & $0.72 * * *$
\end{tabular}

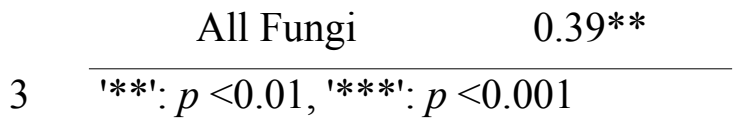

4 